\title{
Visualization by PIV of dynamic stall on a vertical axis wind turbine
}

\author{
Carlos Simão Ferreira · Gijs van Kuik • \\ Gerard van Bussel · Fulvio Scarano
}

Received: 25 February 2008/Revised: 25 June 2008 / Accepted: 4 July 2008/Published online: 3 August 2008

(C) The Author(s) 2008

\begin{abstract}
The aerodynamic behavior of a vertical axis wind turbine (VAWT) is analyzed by means of 2D particle image velocimetry (PIV), focusing on the development of dynamic stall at different tip speed ratios. The VAWT has an unsteady aerodynamic behavior due to the variation with the azimuth angle $\theta$ of the blade's sections' angle of attack, perceived velocity and Reynolds number. The phenomenon of dynamic stall is then an inherent effect of the operation of a VAWT at low tip speed ratios, impacting both loads and power. The present work is driven by the need to understand this phenomenon, by visualizing and quantifying it, and to create a database for model validation. The experimental method uses PIV to visualize the development of the flow over the suction side of the airfoil for two different reference Reynolds numbers and three tip speed ratios in the operational regime of a small urban wind turbine. The field-of-view of the experiment covers the entire rotation of the blade and almost the entire rotor area. The analysis describes the evolution of the flow around the airfoil and in the rotor area, with special focus on the leading edge separation vortex and trailing edge shed vorticity development. The method also allows the quantification of the flow, both the velocity field and the vorticity/circulation (only the results of the vorticity/
\end{abstract}

C. Simão Ferreira $(\bowtie) \cdot$ G. van Kuik · G. van Bussel DUWIND, Wind Energy Section,

Delft University of Technology, Kluyverweg 1,

2629 HS Delft, The Netherlands

e-mail: c.j.simaoferreira@tudelft.nl

\section{F. Scarano}

Faculty of Aerospace, Aerodynamics,

Delft University of Technology, Kluyverweg 1,

2629 HS Delft, The Netherlands circulation distribution are presented), in terms of the phase locked average and the random component.

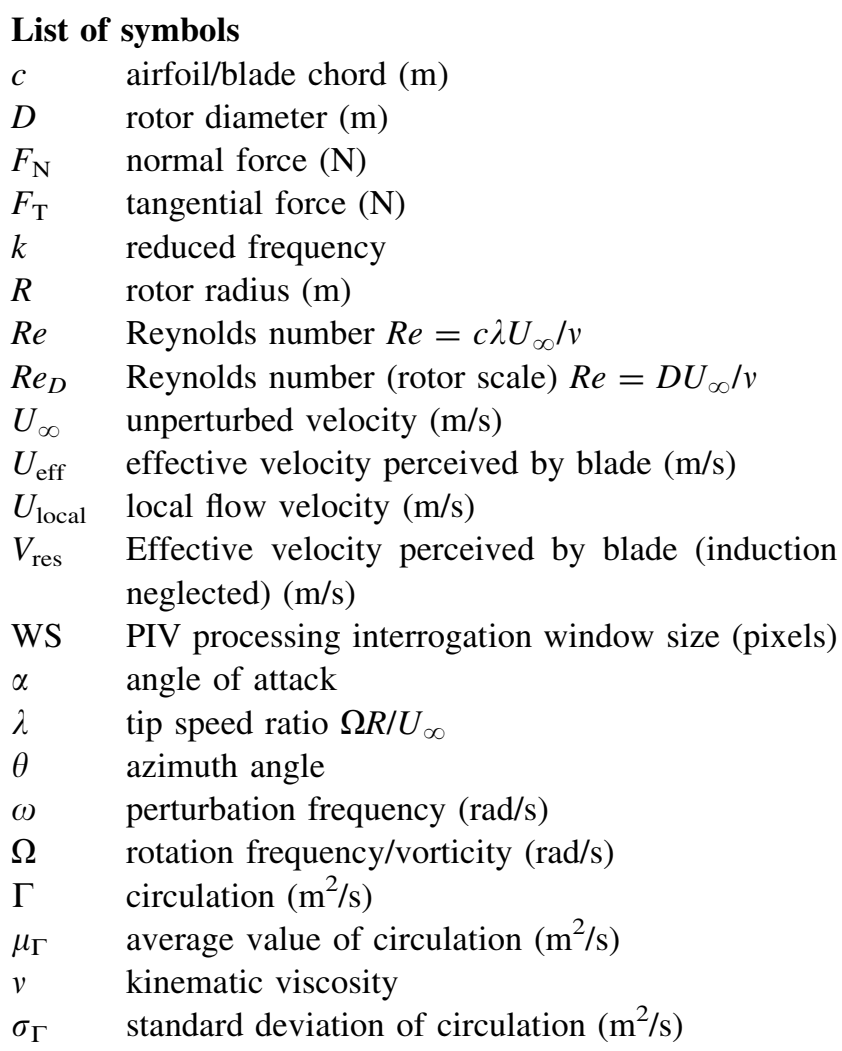

\section{Introduction}

The increasing awareness of the need for environmentally sustainable housing and cities has driven the development of wind energy conversion systems for the built 
environment. One of the results of the development of solutions for the built environment is the reappearance of vertical axis wind turbines (VAWTs). Extensive research on VAWTs was conducted until the end of the 1980s (especially in North America), when, due to the increasing success of the application of horizontal axis wind turbines (HAWTs) in Europe, it was discontinued. Yet, in the built environment, VAWTs present several advantages over the more common HAWTs, namely: low sound emission (a consequence of its operation at lower tip speed ratios), better esthetics due to its three-dimensionality (more suitable for integration in some architectural projects, since it follows the concept of volume of the building), its insensitivity to yaw and its increased performance in skew (see Mertens et al. 2003; Simão Ferreira et al. 2006).

The present work aims at measuring the velocity field with dynamic stall at Reynolds numbers $R e \approx 10^{5}$ and $\lambda=2,3$ and 4 , conditions close to operational values for small VAWTs (below $2 \mathrm{~kW}$ rating) in full-scale urban applications. The tip speed ratio, defined as the ratio between the velocity of the unperturbed flow and the rotational velocity of the rotor, is one of the most important parameters for rotor aerodynamics. As seen in Fig. 1, the effective velocity perceived by the blade $U_{\text {eff }}$ is a combination of the local flow velocity $U_{\text {local }}$ and the velocity due to the movement of the blade $\Omega R=\lambda U_{\infty}$. The local flow velocity $U_{\text {local }}$ is a function of the unperturbed velocity $U_{\infty}$ and the induction generated by the rotor. The tip speed ratio at which the rotor operates, in combination with the local induction, determines the magnitude of the effective

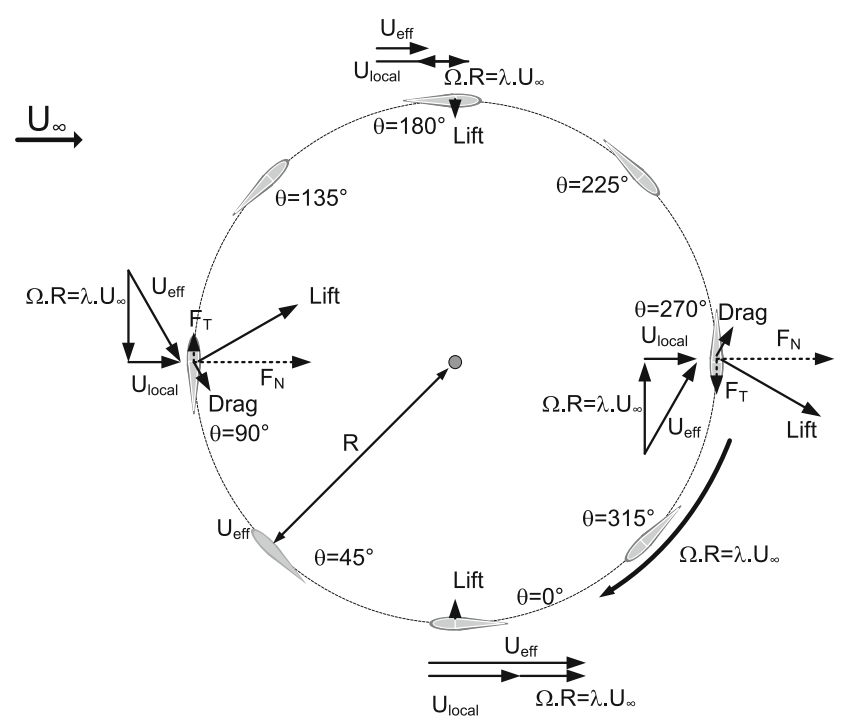

Fig. 1 Scheme of the rotation of a VAWT at eight azimuthal positions; the scheme represents the effective velocity $U_{\text {eff }}$ perceived by the blade at $\theta=0^{\circ}, 90^{\circ}, 180^{\circ}$ and $270^{\circ}$, and the resultant orientation of the Lift and Drag forces and their decomposition in normal $F_{\mathrm{N}}$ and tangential $F_{\mathrm{T}}$ forces velocity and its orientation. This, in turn, determines the orientation of the lift, and thus the tangential force $F_{\mathrm{T}}$, responsible for the generation of torque.

Dynamic stall is an inherent effect of the operation of a VAWT at low tip speed ratios $(\lambda<5)$, with significant impact on both load and power. Dynamic stall occurs when an airfoil, operating in unsteady flow, overcomes the static stall angle (Leishman 2002). For a fixed blade geometry VAWT in unsteady flow, for a given induction factor, the angle of attack is a function of azimuth angle; for $\lambda<5$, the angle of attack can then overcome the static stall angle (Fig. 2), causing dynamic stall. During dynamic stall, large leading edge separated vortices are formed, delaying the occurrence of loss of lift, until they are convected over the surface, resulting in a fast drop in lift. In VAWT, the rotational movement of the blade in relation to the incoming wind adds an extra complexity to the traveling and dynamics of the vorticity over the blade and in the rotor.

The effect of dynamic stall on the evolution of forces on the blade is seen in Fig. 3; the figure shows the nondimensioned tangential force and normal force over three rotations. The forces are simulated with an unsteady Reynolds averaged Navier-Stokes $k-\varepsilon$ improved model of the work presented by Simão Ferreira et al. (2007).

Previous experimental work on dynamic stall effects on VAWTs focused mainly on performance measurements (see Laneville and Vittecoq 1986) or using flow visualization techniques (see Brochier et al. 1986). Fujisawa and Shibuya $(2001,1999)$ developed a first attempt at flow field

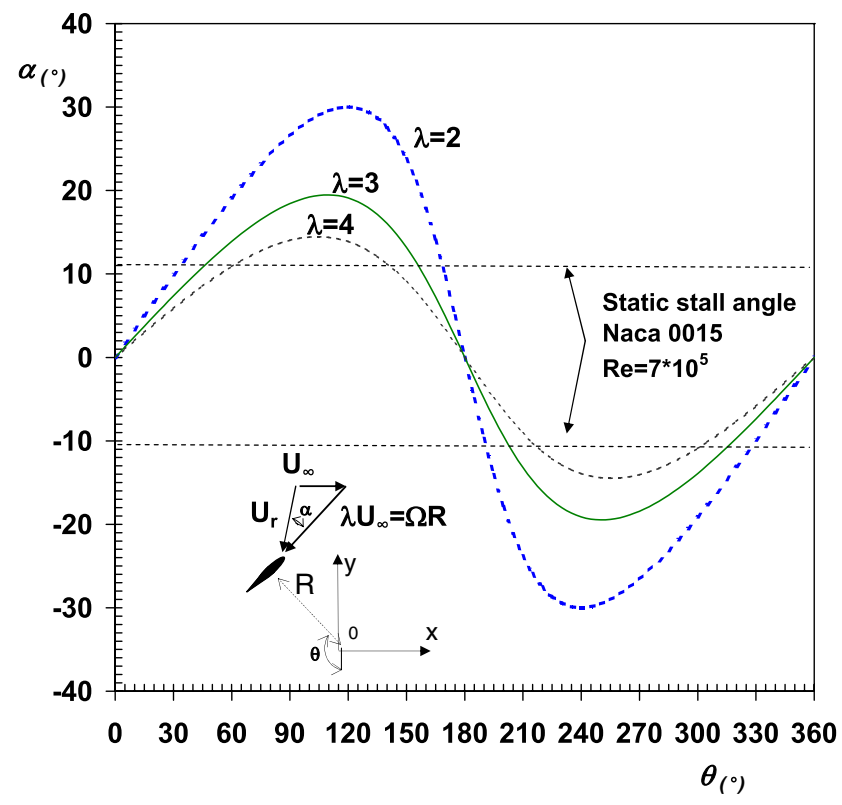

Fig. 2 Plot of the angle of attack perceived by the blade (without induction) for $\lambda=2,3$ and 4 ; the curves are plotted in comparison to the static stall angle at similar $R e$ for a NACA0015 


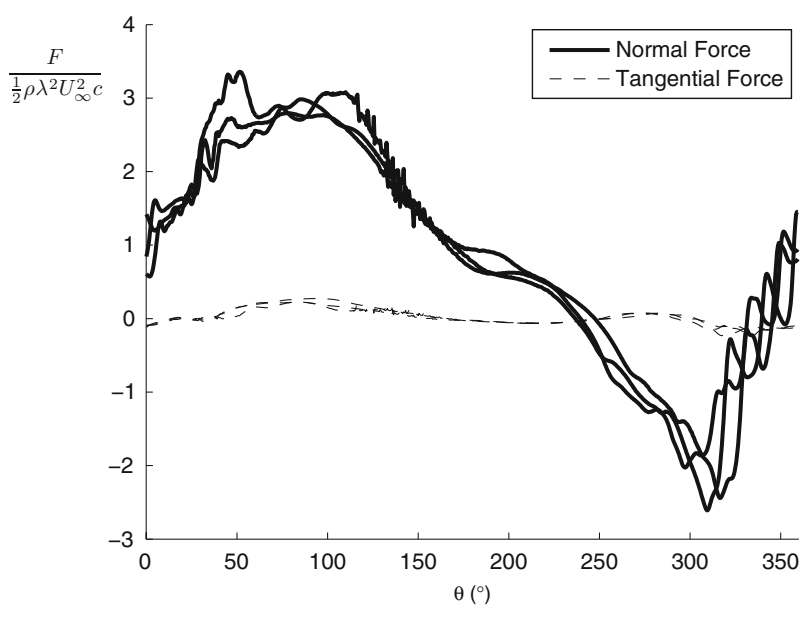

Fig. 3 Plot of the tangential and normal force coefficient as simulated with a URANS $k-\varepsilon$ model, over three rotations

measurements of a Darrieus using particle image velocimetry (PIV) at Reynolds numbers $R e_{D}=3,000$ and $R e=1,000$ (water flow). The significantly lower Reynolds number as compared with full-scale wind energy applications results in a different development of the shedding of vorticity, with larger vortex pairs being shed than at higher Reynolds. The improvement of PIV techniques during the last years allows a revisit of this phenomenon with higher spatial resolution, acquiring not only a better qualitative but also quantitative description of the flow.

The level of unsteadiness is determined by the reduced frequency $k$, defined as $k=\omega c / 2 U_{\text {eff }}$, where $\omega$ is the angular frequency of the unsteadiness, $c$ is the blade's chord and $U_{\text {eff }}$ is the velocity of the blade. In this experiment, due to the variation of $U_{\text {eff }}$ with rotation angle, $k$ was defined for a VAWT as $k=\omega c /\left(2 \lambda U_{\infty}\right)=\omega c /(2 \omega R)=$ $c /(2 R)$, where $\lambda$ is the tip speed ratio and $R$ is the radius of the turbine. For this experimental work $k=0.125$, placing the conditions in the unsteady aerodynamics region.

Experimental data is needed to gain a better knowledge of the VAWT dynamic stall process, and also to obtain an experimental database that can be used to validate the flow field calculated with numerical models; such validation is not possible with force or pressure data alone.

\section{Experimental apparatus and procedure}

\subsection{Wind tunnel facility}

The low-speed low-turbulence wind tunnel (LTT) of the Faculty of Aerospace Engineering of Delft University of Technology (TU Delft) has an octagonal test section that is $1.80 \mathrm{~m}$ wide, $1.25 \mathrm{~m}$ high and $2.60 \mathrm{~m}$ long. This tunnel has a contraction ratio of 17.6 , resulting in a maximum test

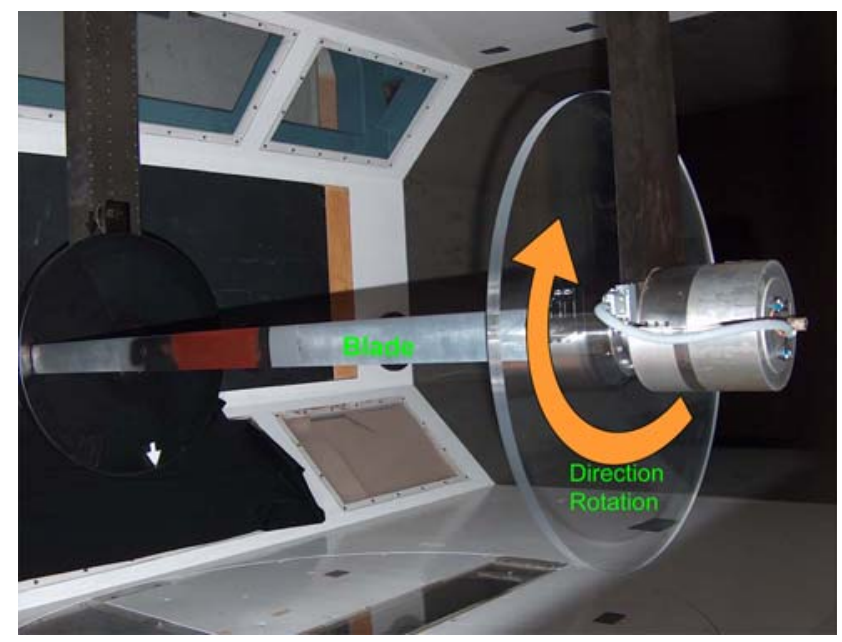

Fig. 4 Rotor model in the test section

section velocity of $100 \mathrm{~m} / \mathrm{s}$ and a low turbulence intensity ranging from $0.015 \%$ at $10 \mathrm{~m} / \mathrm{s}$ to $0.07 \%$ at $75 \mathrm{~m} / \mathrm{s}$.

\subsection{The rotor}

The rotor model is an H-Darrieus vertical axis wind turbine ${ }^{1}$ initially built for the investigation of pitchable VAWTs in ground effect (Coene 1983). The model is redesigned for the PIV experimental work, allowing a better access to the field of view (FOV), adding phase locking control and decreasing laser reflections. The large aspect ratio of the blade $(A R=20)$ and a symmetry disk at each blade tip helps to achieve 2D flow conditions at the mid-section.

The rotor is placed in the center of the test section, with rotor axis at a distance of $0.80 \mathrm{~m}$ from the lower wall of the test section (see Fig. 4). The 2D geometric blockage ratio of the model in the wind tunnel is $32 \%$ at the measured cross section position. However, one should note that for a VAWT most induction is concentrated at the rotor axis height; Navier-Stokes simulations performed by the authors of the experiment have shown that the blockage effect can be neglected for the present analysis.

The rotor is designed as a single bladed NACA0015 airfoil Darrieus (chord $c=0.05 \mathrm{~m}$ ). The use of a single blade creates a simpler flow, reducing the occurrence of blade vortex interaction ${ }^{2}$ and multiple wakes inside the rotor space. The rotor has a diameter $D=0.4 \mathrm{~m}$. As mentioned above, the blade is supported at its extremities in two flat disks. Two extra supports connecting the blade to the rotation axis are

\footnotetext{
$\overline{1}$ The model as been referred in other work as "cyclogiro". The difference between the two nomenclatures is due to application, since it is one and the same aerodynamic concept. In this work the model will be referred to as a Darrieus VAWT.

${ }^{2}$ Blade-vortex Interaction phenomenae still occurs in the downwind portion of the blade rotation.
} 


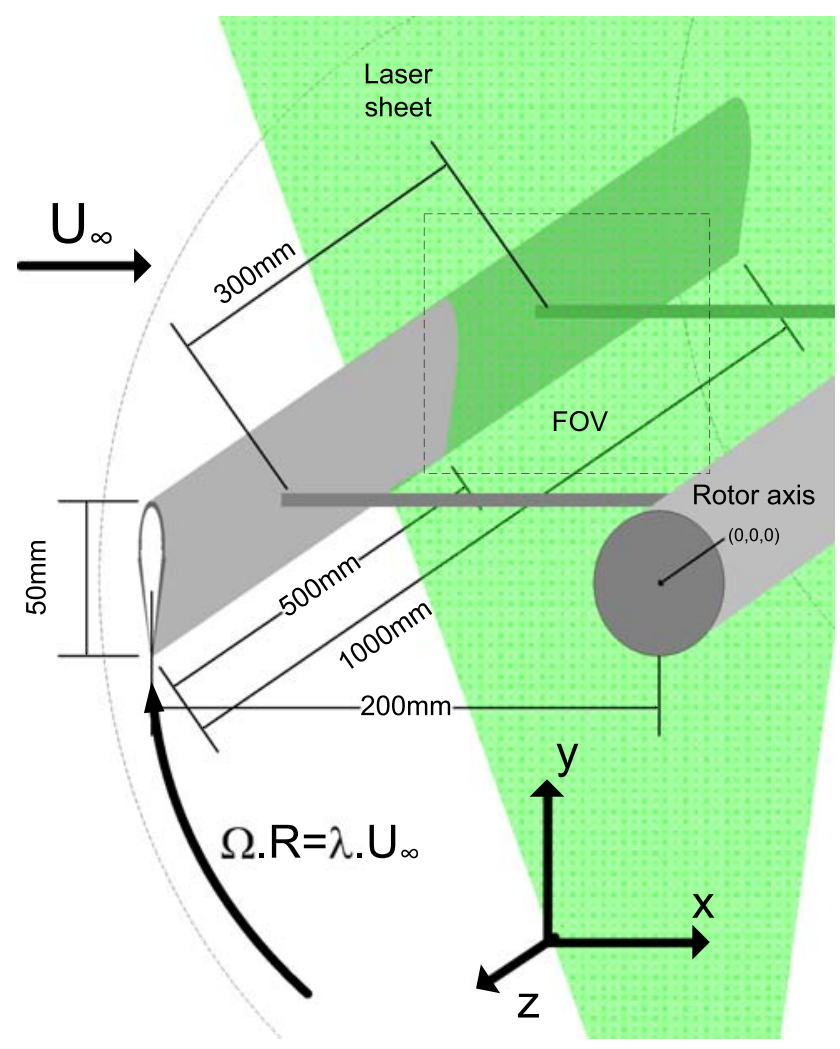

Fig. 5 Schematic of model, detail at field of view (FOV)

added in the cases of higher rotational velocity, in order to prevent deformations of the blade due to centrifugal force. Figure 5 presents a schematic of the geometry of the rotor (view at mid-span/laser sheet location).

The experimental work is performed at Reynolds $R e=5 \times 10^{5}$ and $7 \times 10^{5}$ and tip speed ratio $\lambda=2,3$ and 4. The Reynolds numbers is calculated using the blade's chord and rotational velocity as reference scales; $R e=\frac{R \Omega c}{v}$, where $v$ is the kinematic viscosity of air. Table 1 presents the reference parameters of the different experimental runs.

\subsection{Diagnostic apparatus}

The flow is continuously seeded with approximately $1 \mu \mathrm{m}$ droplets generated by a fog machine. The particle tracers are illuminated by a light sheet introduced vertically into

Table 1 Range of flow and rotor parameters for all experimental runs

\begin{tabular}{lcclll}
\hline$\Omega(\mathrm{rad} / \mathrm{s})$ & $\lambda$ & $U_{\infty}(\mathrm{m} / \mathrm{s})$ & $R e$ & $R e_{\max }$ & $R e_{\min }$ \\
\hline 75 & 2 & 7.5 & $5 \times 10^{4}$ & $7.4 \times 10^{4}$ & $2.5 \times 10^{4}$ \\
75 & 3 & 5.0 & $5 \times 10^{4}$ & $6.6 \times 10^{4}$ & $3.3 \times 10^{4}$ \\
75 & 4 & 3.7 & $5 \times 10^{4}$ & $6.1 \times 10^{4}$ & $3.7 \times 10^{4}$ \\
105 & 2 & 10.5 & $7 \times 10^{4}$ & $1.0 \times 10^{5}$ & $3.5 \times 10^{4}$ \\
105 & 3 & 7.0 & $7 \times 10^{4}$ & $9.2 \times 10^{4}$ & $4.6 \times 10^{4}$ \\
105 & 4 & 5.3 & $7 \times 10^{4}$ & $8.7 \times 10^{4}$ & $5.2 \times 10^{4}$ \\
\hline
\end{tabular}

the test section, perpendicular to the blade and located at it's mid span. The laser light sheet is generated by a Quantec CFR $200 \mathrm{Nd}$ :YAG laser (200 mJ/pulse), and is approximately $2 \mathrm{~mm}$ thick at the FOV. A CCD camera of $1,374 \times 1,040$ pixels is used with a narrowband green filter for daylight interference. The reference FOV is approximately $120 \mathrm{~mm} \times 100 \mathrm{~mm}$; the time interval between exposures is set based to an approximately 8-pixel displacement assuming $U=4 U_{\infty}$. Approximately between 30 and 100 samples are acquired per azimuthal position. The images are analyzed with the iterative multi-grid window deformation technique (Scarano and Riethmuller 2000) with an integration window size of 31 pixels and overlap factor of $50 \%$.

\section{Phase averaging of the velocity field}

The analysis of the flow and dynamic stall development is performed assuming the existence of a dominant phase locked average flow field, determined by the azimuthal position of the blade. The instantaneous flow can be regarded as the result of a mean flow, a phase averaged flow and a random fluctuating term, following the traditional triple decomposition. The predominance and asymmetry of the phase averaged flow, and the importance of the vorticity development in terms of rotor induction, imply that the analysis of a time averaged mean flow is irrelevant for physical insight. Thus, the analysis will focus on the phase averaged and random flow components.

Wernert and Favier (1999) presents an analysis on the phase averaging and convergence of the measured average towards the real average for the case of velocity vectors at a given point (in the airfoil's suction side); from this result it is inferred that an acceptable approximation of the velocity at one point can be obtained with ensembles of a few hundred samples.

The source of this large variability in single point velocity is the complex vortical structure released over the airfoil's suction side. These multiple vortices cause the high randomness in the point velocity, due to both the variability of their strength as well as their spatial distribution and vortex breakdown; the result is that the velocity at any given point inside the vortex region will have a large variability, even if the strength of the total vortex system is fairly constant. Although several hundreds of samples are required to estimate the phase average value of the velocity at a specific point, a reasonable estimate of average strength of the vortex structure can be achieved with a few dozen samples, for most of the flow regions where diffusion of the vortex is yet small. The current work analyzes the flow by focusing on the strength and distribution of vortical structures, instead of individual point velocities. 
Such methodology, analyzing an integral value as the circulation associated with the shed vorticity, has the following advantages:

- it presents a more useful, concise, effective and reproducible parameter for model validation, since it focus on a single integral parameter (in comparison with validation through velocity field),

- it decreases the importance of the randomness of the vortical structures' location/shape, giving more emphasis to the randomness of its magnitude,

- a practical estimation can be achieved using less samples (dozens, instead of hundreds),

- and it allows for a quantification of the magnitude and distribution of the random component.

The use of circulation as the evaluation parameter is problematic in the regions of the flow where the vortex is very diffused, and a small value of vorticity is spread over an large area. For these regions of the low, larger numbers of samples are then required (in the vicinity of a hundred) to compensate for a relatively larger effect of background noise.

\subsection{The tip speed ratio as flow governing parameter}

The experimental work covers three tip speed ratios and two Reynolds numbers. However, the results presented will focus mainly on the case of $\lambda=2$, for it presents the clearest effects of dynamic stall. The cases of $\lambda=3$ and $\lambda=4$ will be presented later in this work for comparison with the results discussed for $\lambda=2$.

\subsection{Determining the phase average and random component of leading edge vortex}

The quantification of the circulation of the vorticity shed at the airfoil surface will focus on the counter-rotating vortex separated at the leading edge, when this is clearly away from the airfoil surface. The quantification of the vorticity close to the surface of the blade is compromised by the reflection of the laser from the surface. For $\lambda=2$, the distance between the vorticity generated at the leading edge and the surface is sufficient to minimize the effect of the surface reflection on the measurements post-processing and allow the quantification of the vorticity magnitude.

Figure 6 shows the phase average (30 samples) vortex structure associated with the leading edge separated vorticity $^{3}$ for $\theta=113^{\circ}$ and $R e=5 \times 10^{5}$. The contour of the

\footnotetext{
3 The remaining vortical structures outside the contour of the vortex are made invisible to simplify the figure. These structures will be discussed later in this paper.
}

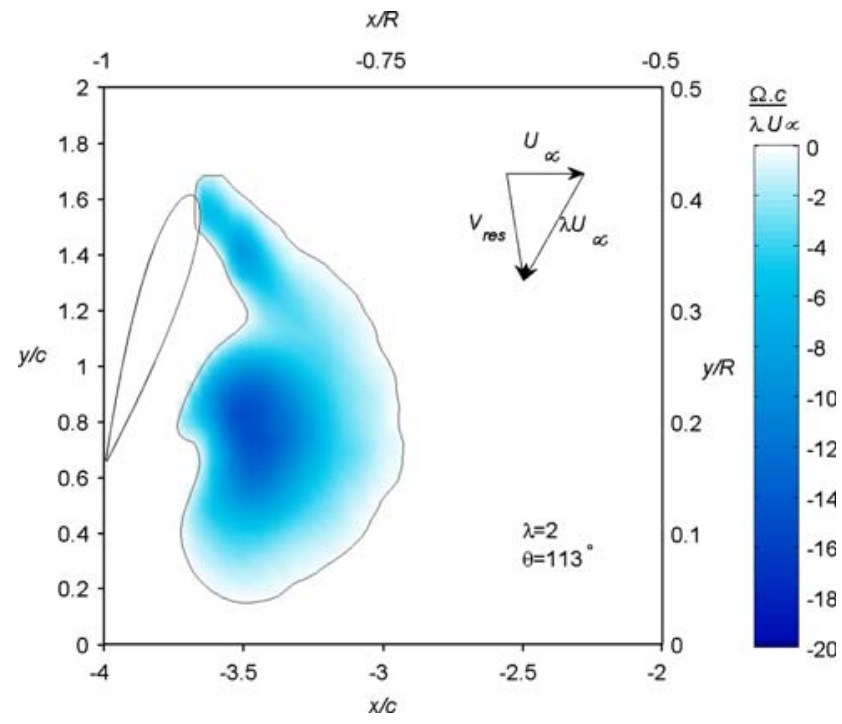

Fig. 6 Leading edge phase average separated vortex for $\lambda=2$, $\operatorname{Re}=50,000, \theta=113^{\circ}$

vortex is determined as the location at which the vorticity goes to zero.

Although the vortical structure resembles a conventional vortex with a central core and radially decreasing vorticity, this is misleading. Figure 7 presents the instantaneous vortical structures for 3 of the 30 instantaneous measurements used to evaluate the phase averaged result. Two main differences exist between the phase average result and the instantaneous results:

- the shape of the null vorticity contour that defines the location of the vortical structures,

- the existence of several smaller vortical structures (darker areas) in the instantaneous results instead of a single large vortical structure as seen in the phase average.

The actual vortical structure shed from the leading edge consists of several connected small vortical structures, varying in magnitude and location in each sample. Although varying, their magnitude and location correspond, in average, to what is observed in the phase locked average, inside its null vorticity contour. Nevertheless, these clockwise vortical structures interact with counterrotating vortical structures generated over the suction side of the blade surface which possess their own randomness (the evolution of this counter-clockwise vorticity is discussed later in the paper).

The averaging of the circulation of the vortical structures is a linear process. Therefore, the difference between the magnitude of the phase locked average leading edge vortex and the average magnitude of the instantaneous vorticity is a result of the averaging at the contour limit. It 
Fig. 7 Instantaneous (three samples) leading edge separated vortex for $\lambda=2, R e=50,000$, $\theta=113^{\circ}$
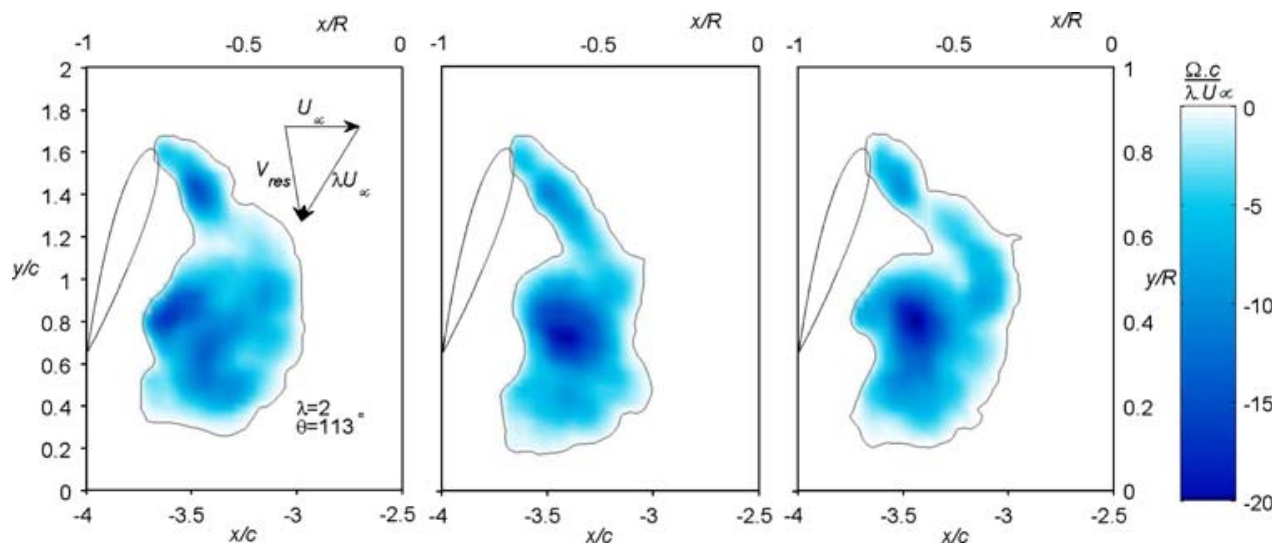

includes both clockwise and counter-rotating vortical structures generated at the blade's surface.

The differences between the values of circulation calculated by both methods indicates the uncertainty related to the low number of samples used. For an infinite number of samples, it is expected that both methods would converge to a single estimate.

Figure 8 shows a comparison of the value of circulation calculated with the phase averaged velocity field, and the average of the values of circulation calculated for each sample used on the phase locked average.

Figure 8 also presents a comparison between the standard deviation of the circulation of the instantaneous samples and the standard deviation of the circulation along the contour defined by the phase average vortex. The error bars indicate the uncertainty interval at a 0.95 confidence level.

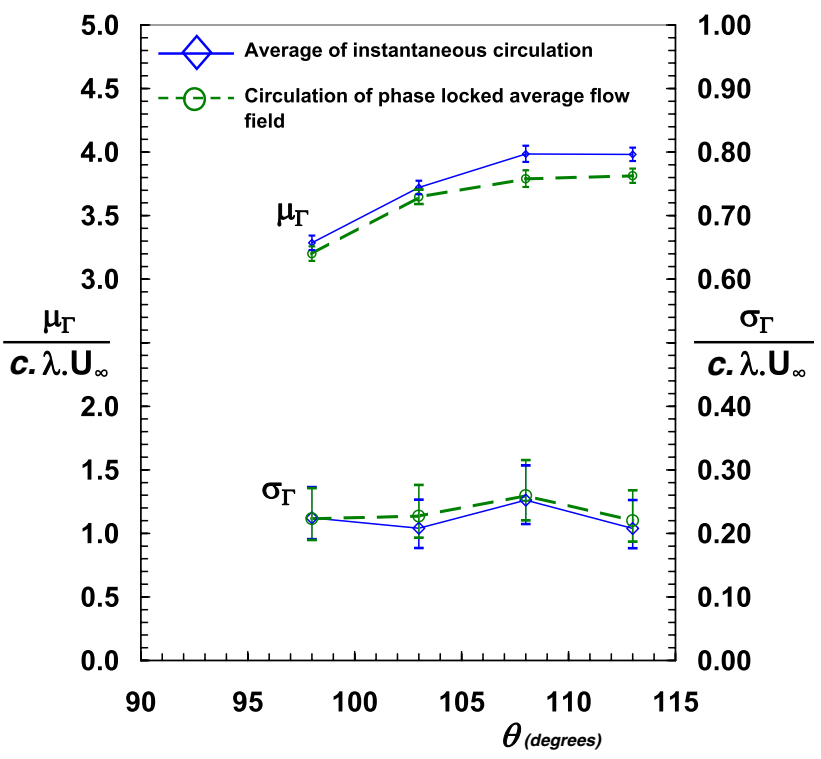

Fig. 8 Comparison average of circulation of individual samples vs circulation of phase averaged flow field, for the leading edge separated vortex at four azimuth angles for $\lambda=2, R e=50,000$. Left circulation; right standard deviation of circulation
The results show a lower value of circulation for the phase averaged case than when one calculates the value of circulation for each individual sample; this is a consequence from the averaging inside the contour of counterclockwise vorticity close to the blade's surface and the definition of the vortex contour, both for the instantaneous samples and the phase averaged flow field. However, the difference is small (approximately $0.05 \Gamma$ in the worst case) and in scale with the error associated with the post-processing methodology or experimental uncertainty.

The results for the estimation of the standard deviation of the circulation of the samples do not show any significant deviation between the two methods of estimation. The circulation of phase average is thus an acceptable approximation for the leading edge separated vorticity average magnitude and its randomness. The randomness of the phase locked average is defined as the standard deviation of the circulation of the instantaneous samples following the contour defined by the phase average.

An assumption of a normal distribution of the circulation for all the samples was used for both estimators (average and standard deviation). The validity of this assumption is shown in Fig. 9, where the normalized distribution of the circulation of the samples for the case $\lambda=2, \theta=113^{\circ}$ is compared with a standard normal distribution. The fitting of the distribution to a normal distribution satisfies a Kolmogorov-Smirnov test at a 0.95 confidence level. This is an important result, since it not only defines the stochastic distribution of the circulation, but also allows testing of the validity of estimators calculated by different models and the determination of the size of the error.

\subsection{Uncertainty associated with the experimental procedure}

The accuracy of the phase locking is usually an important source of error in similar experiments. From the experimental data it is possible to conclude that any oscillation of 


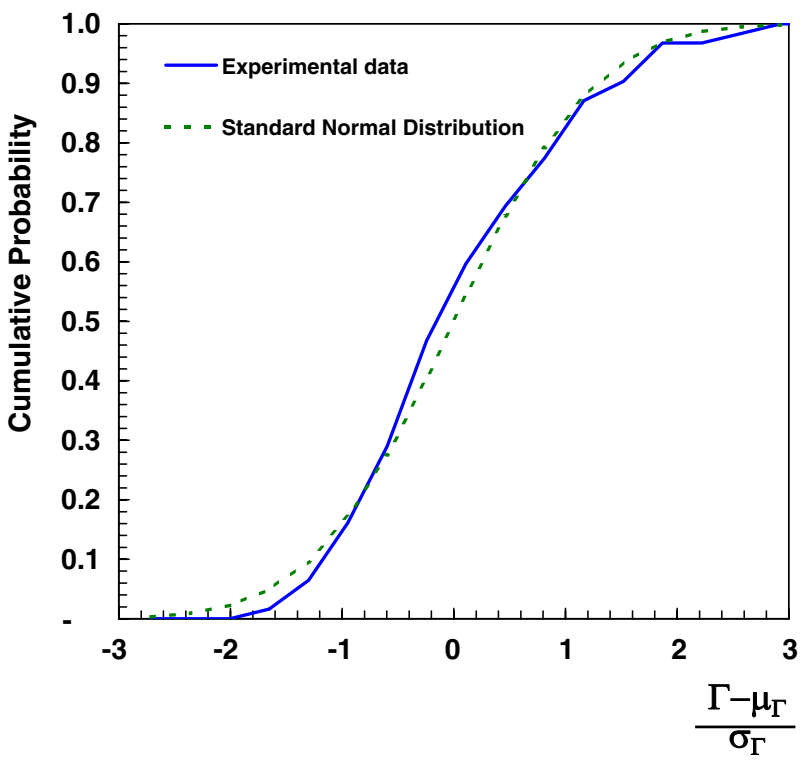

Fig. 9 Probability distribution of the circulation of leading edge separated vortex for $\lambda=2, R e=50,000, \alpha=113^{\circ}$ and comparison with a standard normal distribution)

the phase locking azimuthal angle of the different samples is imperceptible and much smaller than the uncertainty of the actual value of the phase locking azimuthal angle, estimated at $\pm 0.03^{\circ}$. This oscillation is the result of variations during the rotation of the aerodynamic torque and reaction of the controller for torque at the generator. This low level of uncertainty in the azimuthal position can be considered as negligible.

The bias associated with the angle of attack of the blade (due to uncertainty in the definition of zero position and due to blade bending), was estimated at a maximum of $\pm 0.25^{\circ}$, based on observations at the most upwind position of the rotation.

\subsubsection{Wind tunnel blockage}

The problem of the influence of the blockage on rotor experiments has been a topic of research for almost a century (see Glauert 1935; Loeffler and Steinhoff 1985; Mikkelsen and Sørensen 2002; Mikkelsen 2003). Still, these models focus on horizontal axis rotors (both propellers and wind turbines), where the rotor is modeled as an actuator disk of constant induction over the entire disk. In the case of a VAWT, the load and induction is not uniform over the rotor area; it usually presents a sinusoidal distribution, with a peak around $y=0 \mathrm{~mm}$. The blockage effect of a VAWT is thus much smaller than what is calculated with the constant induction assumption used by Glauert (1935). To assess the extent of the blockage effect, a 2D Navier-Stokes numerical model is used (see Simão Ferreira et al. 2007) to simulate the case of the rotor in the wind tunnel and compare with the simulation of the same VAWT in open field. The difference in thrust between the two cases is negligible; thus blockage effects are considered to be small.

\subsection{Influence of the size of the interrogation window}

Another source of uncertainty in the data processing step is an incorrect choice of the interrogation window, the subframe pixel area where the displacement is evaluated. In the current experimental work this is particularly severe due to the large velocity gradient in the separated flow region over the suction side of the airfoil. A too large interrogation window will average the velocity field, while a too small one will not contain enough particles and a good noise to signal ratio.

Figure 10 presents the value of circulation of the leading edge vortex for $\lambda=2$ calculated using three different sizes of WS, corresponding to scales of $0.05 c, 0.07 c$ and $0.1 c$, with adaptive spatial resolution (see Scarano and Riethmuller 2000), with $50 \%$ overlapping. Notice that the average values differ mainly for azimuthal angles between $104^{\circ}$ and $130^{\circ}$, where the developing leading-edge vortex strongly interacts with the counter-clockwise vorticity generated between the vortex and the suction side surface of the airfoil. This difference disappears for the rest of the (observed part of the) rotation where the shape of the vortex is such that it no longer strongly interacts with the opposite vorticity close to the blade surface, just before full separation of the vortex. The considered setting of WS of $0.07 c$ (31 pixels) will tend to average out some of the

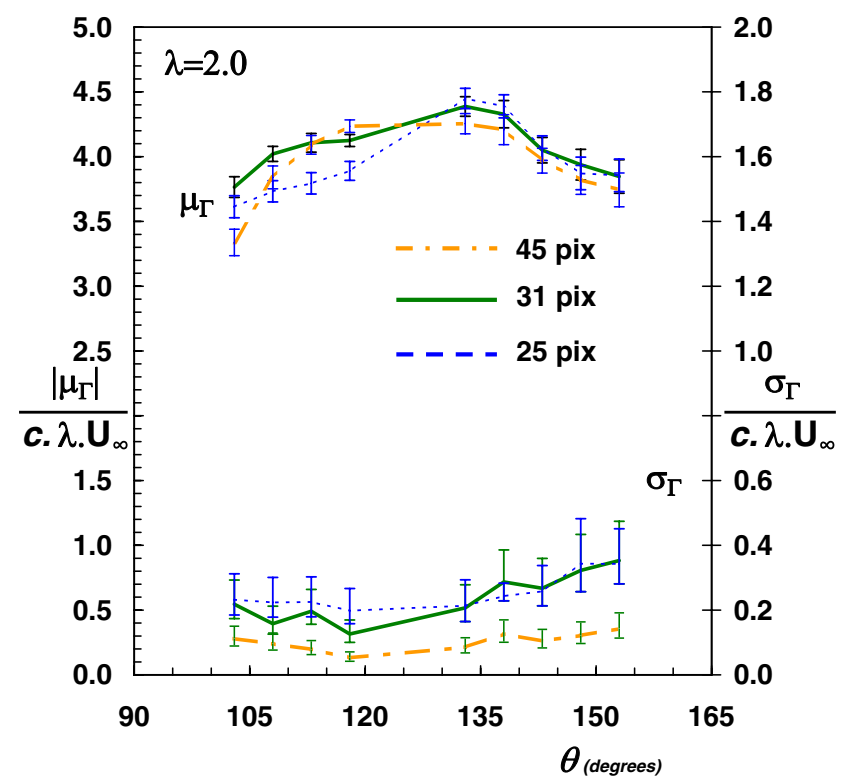

Fig. 10 Calculated leading edge vortex circulation and standard deviation for two interrogation window sizes over part of the rotation 
vorticity at the interaction, thus resulting in a possible overestimation of the value of circulation; yet, the large velocity gradients lead to lower particle count, resulting that using smaller interrogation windows decreases the signal-to-noise ratio which induce larger errors.

The standard deviation of the measured circulation does not show any significant difference for the two window sizes because it is mainly determined by the strong vorticity at the core. Once again, the error bars in the measurement points relate to the uncertainty of the estimators at a 0.95 confidence level; they do not represent the experimental error.

\section{The influence of Reynolds numbers}

The original intent in performing the experiment at different Reynolds numbers is to evaluate its effect on the behavior of the flow. Due to limitations in the structure of the model, the maximum Reynolds number could exceed $R e=7 \times 10^{5}$. The dynamic stall process is dominated by the leading edge separated laminar flow, and no visible difference is perceived between the experimental results at the two Reynolds numbers. Figure 11 shows the measured non-dimensionalized circulation of the leading edge vortex for $\lambda=2$ for both Reynolds numbers; the resulting flow presents the same behavior (this is confirmed by other measurements not presented here). At other $\lambda$ the differences in flow behavior were also smaller than the measurement accuracy of the experimental setup. Thus, the results are not discussed in relation to Reynolds number, but only in relation to tip speed ratio $\lambda$.

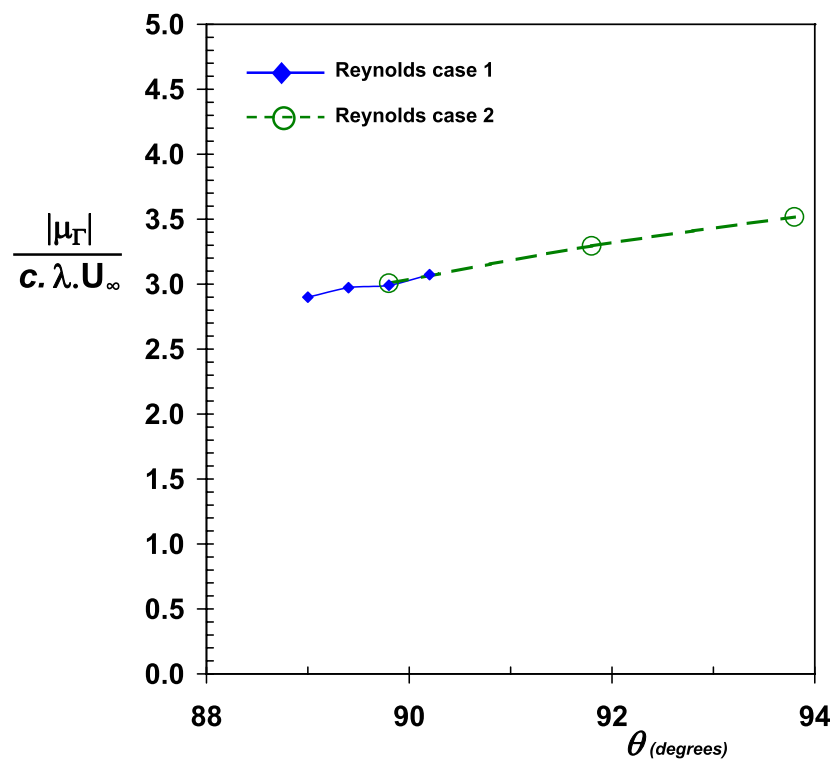

Fig. 11 Comparison of the value of circulation of the leading edge vortex in the vicinity of the $90^{\circ}$ position for two Reynolds numbers

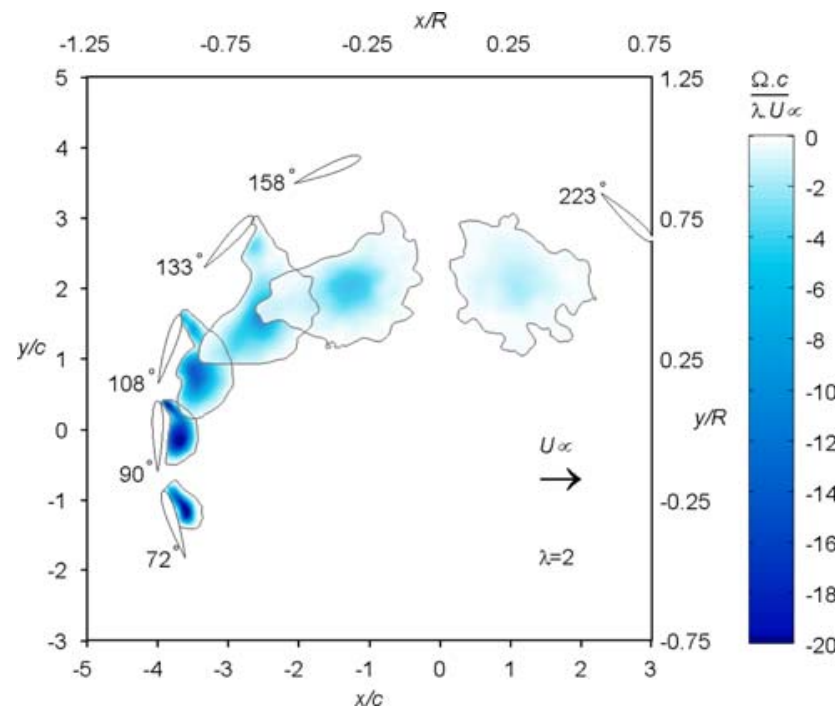

Fig. 12 Evolution of the circulation of leading edge separated vortex for $\lambda=2$ at $90^{\circ}, 108^{\circ}, 133^{\circ}$ and $158^{\circ}$

\section{Variation of shed vorticity with rotation angle}

Shed vorticity is used as the main parameter to describe the flow over the rotation of the blade; its selection over the direct presentation of the velocity field is due to two main characteristics:

- the shed vorticity is confined to a limited portion of the field of view, thus allowing a more focused analysis, both in position and value,

- the shed vorticity allows for timeline analysis of the development of the flow and a direct relation between the flow field and the loads in the blade.

The current work presents two forms of analysis:

- a description of the development of the flow and quantification of the shed vorticity field for the three different tip speed ratios,

- and the quantification of the circulation of the leading edge vortex for the $\lambda=2$ case.

\subsection{The $\lambda=2$ case}

Figure 12 presents the evolution of the clockwise leading edge vortex at six moments defined by the blade's azimuthal position ${ }^{4}$. These six moments cover the development of the leading edge laminar separation vortex $\left(\theta=72^{\circ}\right)$, its growth over the suction side of the airfoil until it reaches the full chord length $\left(\theta=90^{\circ}\right)$, its convection away from the suction side of the airfoil $\left(\theta=108^{\circ}\right.$

\footnotetext{
4 The remaining vorticity outside the vortex contour is made invisible for clarity of the figure
} 


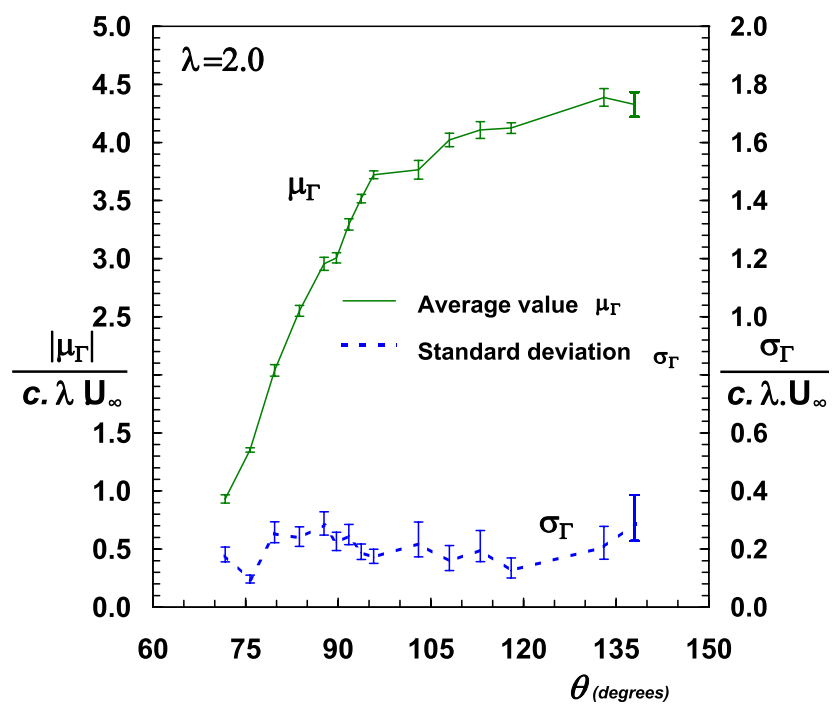

Fig. 13 Evolution of the circulation of leading edge separated vortex for $\lambda=2$

and $\theta=133^{\circ}$ ) and its release from the airfoil, subsequent convection and diffusion $\left(\theta \geq 138^{\circ}\right)$.

Figure 13 shows the evolution of the magnitude of the leading edge vortex from close to its formation until its release at $\theta>138^{\circ}$. The evolution presents two different regions:

- from $\theta \approx 70^{\circ}$ to $\theta \approx 95^{\circ}$, where there is an almost linear growth of the magnitude of the leading edge vorticity,

- from $\theta \approx 95^{\circ}$ to $\theta \approx 138^{\circ}$, where the magnitude continues to grow at a lower rate until it reaches a maximum just before the vortex separates from the airfoil.

The termination of linear increase of magnitude at $\theta \approx 95^{\circ}$ is coincident with the roll up of the trailing edge vorticity (this is discussed later in this paper).

At the detachment of the vortex after $\theta=138^{\circ}$, part of the leading edge vorticity reattaches over the surface of the airfoil (observed in experimental data); this explains the sudden decrease of the amount of vorticity between $\theta=138^{\circ}$ and $\theta=143^{\circ}$ (Figs. 13, 14). The detached vortex experiences diffusion and dissipation of its vorticity (Figs. 12, 14).

\section{Evolution of counter-clockwise vorticity}

To understand the growth rate of the leading edge vorticity in the vicinity of $\theta \approx 95^{\circ}$, it is necessary to observe the evolution of the counter-clockwise vorticity shed at the trailing edge; this vorticity is generated both on the

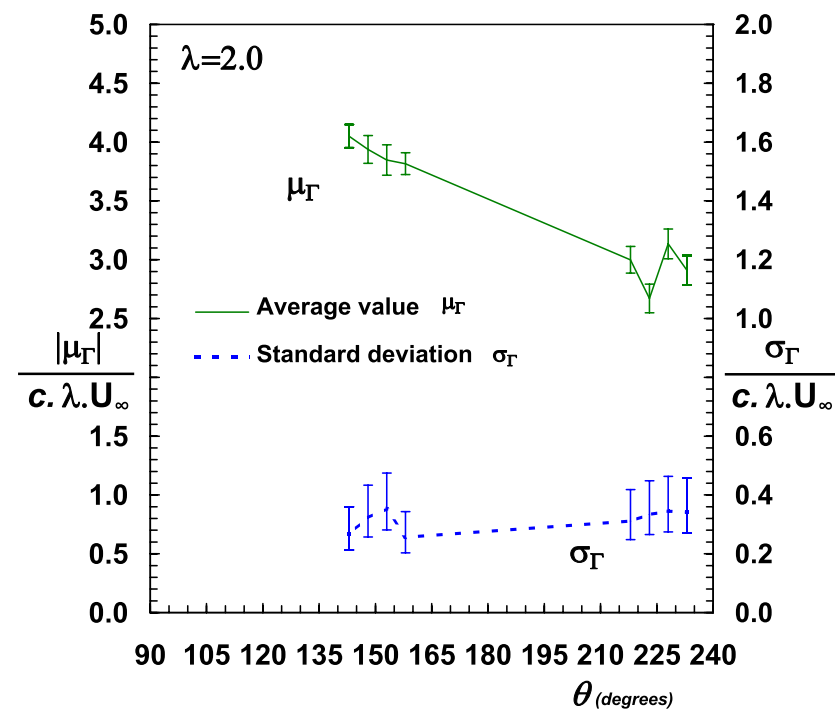

Fig. 14 Evolution of the circulation of the vortex generated at the leading edge, after separation from the airfoil, $\lambda=2$

pressure side and on the suction side of the airfoil, in the section aft of the leading edge vortex separation location (Figs. 15, 16, 17).

Figure 15 presents the post trailing edge wake segment at $\theta=83^{\circ}$. Two adjacent regions of vorticity can be observed: a clockwise (negative) vorticity originated from the leading edge separated vorticity and a counter-clockwise (positive) vorticity generated by the suction side boundary layer, aft of the leading edge separation. As previously seen, the clockwise vorticity will roll-up into a large leading edge vortex (Figs. 16, 17); a detail of this

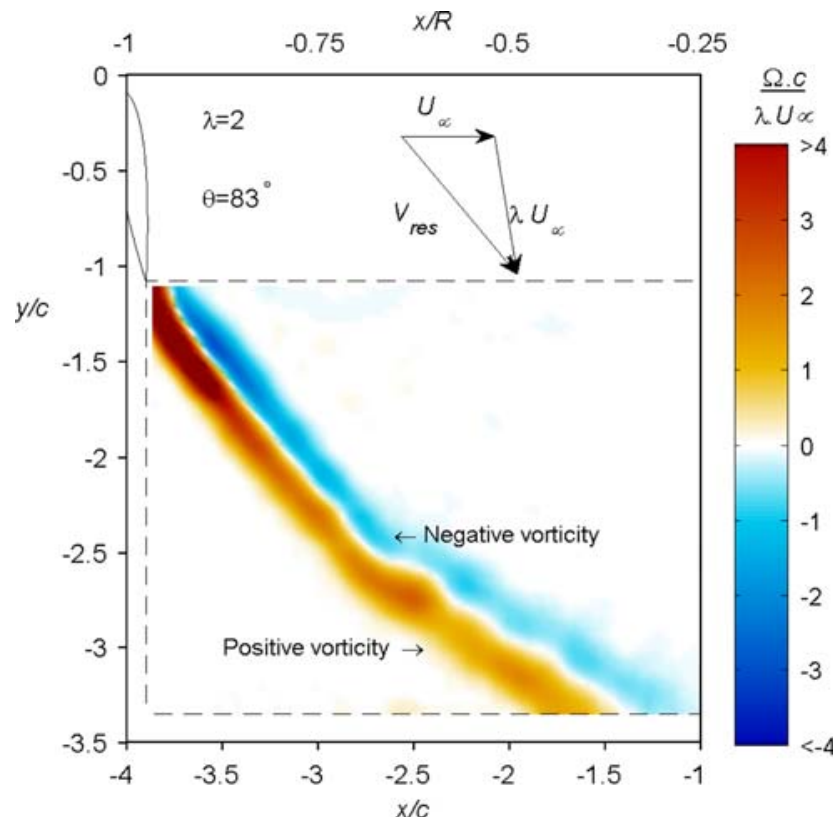

Fig. 15 Vorticity of wake shed at the trailing edge $\left(\theta=83^{\circ}, \lambda=2\right)$ 
Fig. 16 Rolling up of trailing edge vorticity $\left(\theta=90^{\circ}\right.$ and $\theta=98^{\circ}, \lambda=2$ )
Fig. 17 Rolling up of trailing edge vorticity $\left(\theta=104^{\circ}\right.$ and $\left.\theta=110^{\circ}, \lambda=2\right)$
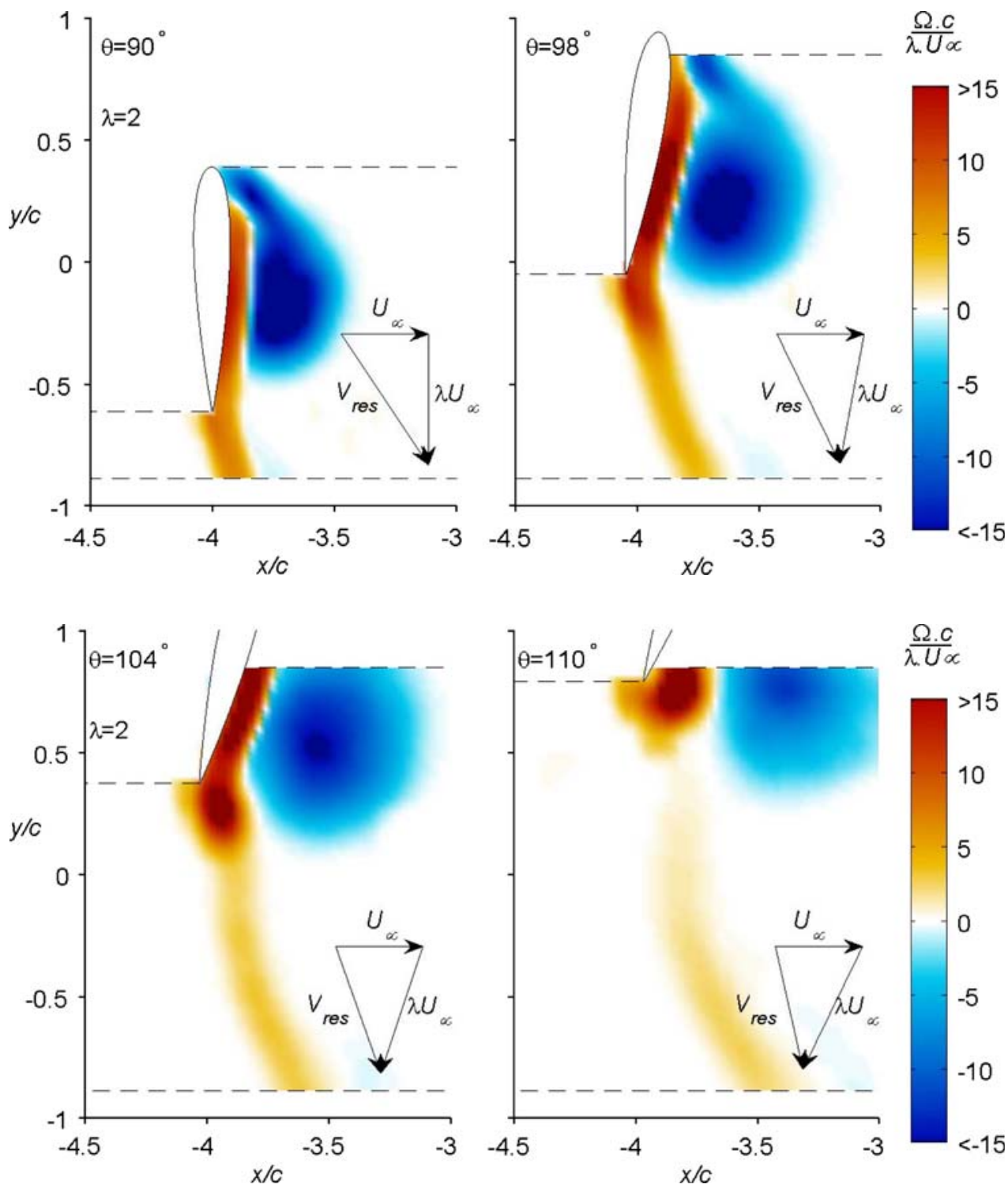

process can be seen in the left half of Fig. $16\left(\theta=90^{\circ}\right)$, where the leading edge vortex and the extremity of the vorticity convected in the remaining wake (identified as 'negative vorticity' in Fig. 15) are presented. As was the case with the clockwise vorticity, the counter-clockwise vorticity also rolls-up, concentrating at suction side/trailing edge. The flow (second half of Fig. 17, $\theta=90^{\circ}$ ) results in two disconnected wake regions: one over the airfoil, consisting of rolled-up vorticity and another of previously shed wake vorticity, containing both negative and positive vorticity. This phenomenon is coincident with the slowing of the increase of the magnitude of the leading edge vorticity at $\theta \approx 95^{\circ}$ mentioned in the previous section.

Figure 18 shows the evolution of the counter-clockwise vorticity shed from the airfoil after its rolling up. Contrary to what happens to the clockwise vorticity shed from the leading edge, the counter-clockwise vorticity shed from the trailing edge is continuous and does not experience any discontinuity when shedding. The vorticity is dragged along with the airfoil, resulting in a blade vortex interaction (BVI) at the downwind passage of the blade. The continuity of the vorticity results from the change of suction/ pressure side of the airfoil as it passes from the upwind part of the rotation to the downwind part. Thus, the side of the airfoil facing the axis of rotation sheds vorticity in the same direction, from the moment the leading edge starts to roll up vorticity in the upwind part of the rotation until the BVI during the downwind part of the rotation. Numerical simulations show that the BVI phenomena affect the pressure distribution and forces in the downwind passage of the blade. Due to the reduced frequency of the flow and the large amplitude of angles of attack, simulations show that an hysteresis effect cause the evolution of the forces in the downwind passage to affect also the upwind passage 


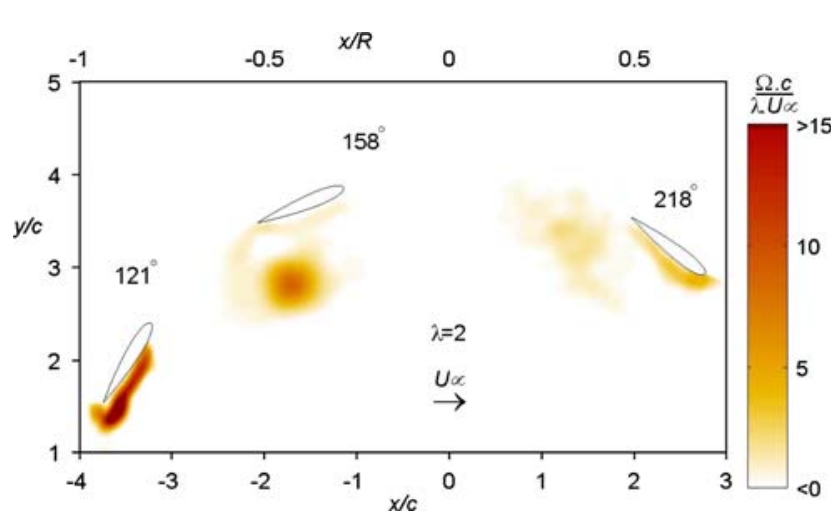

Fig. 18 Evolution of the counter-clockwise vorticity shed after the roll-up of the trailing edge vorticity, $\lambda=2$

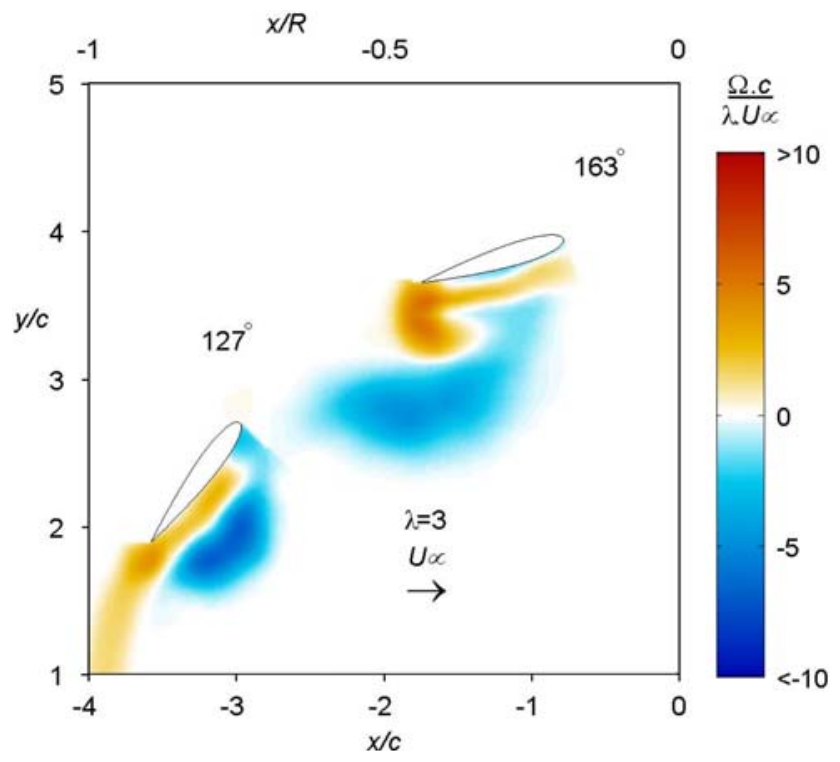

Fig. 19 Shed vorticity at $\theta=127^{\circ}$ and $\theta=163^{\circ}$ for $\lambda=3$

forces. Future experimental work will aim at using PIV data to determine the forces during the full rotation, and determine the full extent of the effect of the BVI.

\section{$7 \lambda=3$ and $\lambda=4$}

The cases for $\lambda=3$ and $\lambda=4$ are succinctly presented for comparison with the $\lambda=2$ case.

For $\lambda=3$, the phenomenons observed in the $\lambda=2$ case once again occur: roll-up of the leading edge clockwise vorticity, followed by the trailing edge counter-clockwise vorticity roll-up, leading to the detachment of the leading edge vorticity. The differences from the $\lambda=2$ case lie in the azimuthal position at which these events occur (later in the $\lambda=3$ case than in $\lambda=2$ ) and the magnitude of the vorticity shed. These differences can be related to the lower

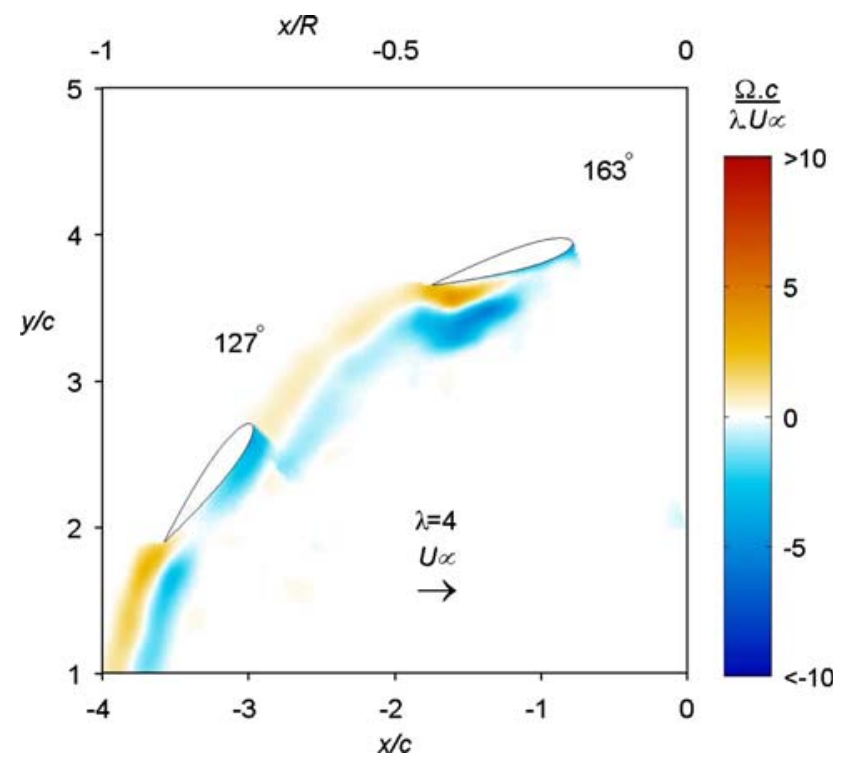

Fig. 20 Shed vorticity at $\theta=127^{\circ}$ and $\theta=163^{\circ}$ for $\lambda=4$

angles of attack occurring at the higher tip speed ratios (Figs. 19, 20).

The lower angles of attack for $\lambda=4$ result in no large roll-up of vorticity or vortex detachment, leading to the development of a continuous wake.

\section{Conclusions}

The particle image velocity results of the evolution of dynamic stall for a Darrieus VAWT at low tip speed ratios identify 2D phase locked and random components of the flow field. The measurements allow the quantification of both the phase average circulation and the random variation of circulation about that phase average of the clockwise vorticity shed from the leading edge laminar separation. This variation about the phase average is wellrepresented by a normal distribution.

The counter-clockwise vorticity generated and shed at the trailing edge is measured, showing the evolution of the shed vorticity, its roll-up, release and subsequent interaction with the downwind passage of the blade. The quantification of the circulation of this vorticity is not possible because a large amount of that vorticity is located close to the surface of the airfoil, where reflection of the laser invalidates the measurement.

The results focus on the case $\lambda=2$, for this presents the largest angles of attack and shedding of the largest amounts of vorticity, thus allowing a clearer quantification or the process. However, the cases $\lambda=3$ and $\lambda=4$ are also observed; comparing the three tip speed ratio cases shows that the different development of the flow is not only dependent on the magnitude of angles of attack, but also on 
how the shed vorticity is transported and continues to interact with the airfoil.

The work also evaluates the phase averaging process and the error associated with it, both in determining the average value of circulation and in determining its randomness. The analysis also shows that the phase average, although reasonably describing magnitude, tends to smooth and present the vorticity in large unique vortices, while the instantaneous samples showed a distribution of several small vortices. These results have implications for the validation of numerical models; although a correct magnitude of shed vorticity may be predicted by the numerical model, its distribution and location of that shed vorticity will result in different flow properties over the surface of the airfoil/blade.

The decision to analyze the vorticity of the flow instead of the actual velocity field is proved useful. The vorticity field is easier to visualize and compare than the velocity field, and it also gives a more direct understanding of the evolution of the flow because it is easier to associate the evolution of lift in the airfoil/blade with the vorticity shed. Also, the phase averaging of the circulation of the vorticity field requires a lower number of samples for a given level of uncertainty than in case the measurement of point velocities.

The results are useful for the validation of the numerical models; they not only give a reasonable description of part of the development of the dynamic stall process, they also present a useful estimate of the strength of the shed vorticity. Yet, the sources of uncertainty of the results must be taken into account in the numerical model validation, specially when using the quantification of the vortex strength.

Although the data has a considerable uncertainty associated, it is currently used by the authors for the validation of CFD models, with promising results to be published. The results are also used for the evaluation of the feasibility of using PIV data for load estimation, which is a follow up of the present work.

Open Access This article is distributed under the terms of the Creative Commons Attribution Noncommercial License which permits any noncommercial use, distribution, and reproduction in any medium, provided the original author(s) and source are credited.

\section{References}

Brochier G, Fraunie P, Beguier C, Paraschivoiu I (1986) Water channel experiments of dynamic stall on darrieus wind turbine blades. J Propuls 2-5:445-449

Coene R (1983) Elements of the aerodynamic theory of cyclogyro wing systems with concentrator effects. Technical Report LR338, Delft University of Technology, Aerospace faculty

Fujisawa N, Shibuya S (2001) Observations of dynamic stall on darrieus wind turbine blades. J Wind Eng Ind Aerodyn 89(2):201-214

Fujisawa N, Takeuchi Y (1999) Flow visualization and piv measurement of floow field around a darrieus rotor in dynamic stall. J Vis $1-4: 379-386$

Glauert H (1935) Aerodynamic theory. Airplane propellers, vol 4. Springer, Heidelberg

Laneville A, Vittecoq P (1986) Dynamic stall-the case of the vertical axis wind turbine. J Solar Energy Eng 108:140-145

Leishman JG (2002) Challenges in modelling the unsteady aerodynamics of wind turbines. Wind Energy 5(2-3):85-132

Loeffler A, Steinhoff J (1985) Computation of wind tunnel wall effects in ducted rotor experiments. J Aircr 22(3):188-192

Mertens S, van Kuik G, van Bussel G (2003) Performance of a h-darrieus in the skewed flow on a roof. J Solar Energy Eng 125:433-440

Mikkelsen R (2003) Actuator disk methods applied to wind turbines. Ph.D. thesis, Technical University of Denmark

Mikkelsen R, Sørensen JN (2002) Modelling of wind tunnel blockage. In: Proceedings of CD-ROM global windpower conference and exhibition

Scarano F, Riethmuller M (2000) Advances in iterative multigrid PIV image processing. Exp Fluids 29:S51-S60

Simão Ferreira C, Bijl H, van Bussel G, van Kuik G (2007) Simulating dynamic stall in a VAWT: modeling strategy, verification and validation with particle image velocimetry data. J Phys Conf Ser 75

Simão Ferreira C, van Bussel G, van Kuik G (2006) Wind tunnel hotwire measurements, flow visualization and thrust measurement of a VAWT in skew. In: AIAA/ASME wind energy symposium

Wernert P, Favier D (1999) Considerations about the phase averaging method with application to ELDV and PIV measurements over pitching airfoils. Exp Fluids 27(6):473-483 